University of Wollongong

Research Online

Faculty of Engineering - Papers (Archive)

Faculty of Engineering and Information

Sciences

$1-1-2006$

\title{
A microslip model of the bonding process in ultrasonic wire bonders Part I: Transient response
}

C M. Hu

Nanyang Technological University, Singapore

N Guo

Nanyang Technological University, Singapore

$\mathrm{H} \mathrm{Du}$

University of Wollongong, hdu@uow.edu.au

W H. Li

University of Wollongong, weihuali@uow.edu.au

M Chen

Nanjing Normal University

Follow this and additional works at: https://ro.uow.edu.au/engpapers

Part of the Engineering Commons

https://ro.uow.edu.au/engpapers/4856

\section{Recommended Citation}

$\mathrm{Hu}, \mathrm{C}$ M.; Guo, N; Du, H; Li, W H.; and Chen, M: A microslip model of the bonding process in ultrasonic wire bonders Part I: Transient response 2006, 860-866.

https://ro.uow.edu.au/engpapers/4856

Research Online is the open access institutional repository for the University of Wollongong. For further information contact the UOW Library: research-pubs@uow.edu.au 


\title{
A microslip model of the bonding process in ultrasonic wire bonders Part I: Transient response
}

\author{
Received: 14 September 2004 / Accepted: 1 February 2005 / Published online: 24 March 2006
}

(C) Springer-Verlag London Limited 2006

\begin{abstract}
Ultrasonic wire bonders with precision capillary tips are widely used for bonding electrical wires to IC chips and circuits. The industry is driving wire-bonding technology towards increased yields, decreased pitch, and ever decreasing cost. However, many technical and material issues will be involved in achieving these goals, such as bond quality monitoring, new failure modes, reliability problems in new plastic mold compounds, and increasing wire sweep problems, in particular lack of the quantitative understanding and validated mathematical model of the bonding process. In this paper, a microslip model is proposed for the bonding process based on the bonding pattern observed, i.e. the wire is bonded at the periphery while the centre is left unbonded. A bilinear hysteresis restoring force is assumed in the microslip model because of its simplicity. The transient displacement response is calculated, and its pattern shows very good agreement with the experiment.
\end{abstract}

Keywords Bilinear $\cdot$ Bonding process $\cdot$ Hysteresis $\cdot$ Microslip · Ultrasonic wire bonder

\section{Introduction}

The ultrasonic wire bonders with precision capillary tips are widely used for bonding wires to IC chips and circuits. U1trasonic bonding is a relatively low temperature process using a combination of pressure and ultrasonic vibration to form a cold weld between two materials. For ultrasonic welding of small specimens such as integrated circuits, the bonding equipment uses

C.M. Hu · N. Guo · H. Du · W.H. Li

School of Mechanical and Aerospace Engineering,

Nanyang Technological University,

Singapore 639798

E-mail: mnqguo@ntu.edu.sg

Tel: 67904488

Fax: 67911859

M. Chen

Department of Control Science and Engineering,

Nanjing Normal University,

Nanjing 210042, P.R. China vibration at high frequency, e.g. 60 or $100 \mathrm{kHz}$. Currently, the industry is driving wire-bonding technology towards increased yields, fine pitch and lower cost [1].

However, there are many technical and material issues involved in achieving these goals. For example, bond quality monitoring, new failure modes, reliability problems in new mould compounds, and increasing wire sweep problems, in particular, a lack of a quantitative understanding of the bonding mechanism. The above issues result in an expanding, but technically challenging future for this method of chip interconnection. There were extensive studies on the mechanism of ultrasonic bonding but they were mostly empirical-based. In general, three approaches had been used to investigate the mechanism of the bond formation. The first one was based on an intuitive concept of a thermal bond developed through frictional heat. The second one considered the experimentally measured movements of the wedge and wire during bonding $[2,3]$. The third one concentrated on an explanation of the observed characteristics of the bond formed $[4,5]$.

Experimental studies had revealed that interfacial temperatures of the ultrasonic bond zone which was measured by the thermocouple method, reached only $70-80^{\circ} \mathrm{C}$. In view of the saturation in thermocouple output and the high mass ratio of interfacial to total-material, localised heating is not expected to be much higher [3]. These temperatures were too low for the ultrasonic bond to be considered a thermally activated diffusion process. In fact, the major portion of the wire deformation was not associated with the machine load alone, but occurred only when the ultrasonic energy was applied.

The ultrasonic pulse is normally applied along the axis of the wire for a short period of time (e.g. 30 to $50 \mathrm{~ms}$ ). During the pulse, parts being bonded must be held firmly enough to allow the wedge movement to be transmitted through the wire to the bond interface. Studies of material movement during bonding had shown that the wire position remains fixed relative to the substrate. Therefore, the wire was not physically scrubbed across the substrate. Measurement using a laser interferometer [6] indicated that a stable wedge motion was being imposed during bond area growth. Values of total wedge displacements for normal bonding varied from 40-200 $\mu \mathrm{in}$, changing with the power setting. 
Therefore, the wedge had a stable motion while the wire did not slide relative to the substrate during the bonding procedure. The friction or sliding model became implausible. In addition, the sliding model predicted preferential bonding at the centre of the interface while the normal ultrasonic bond pattern had a toroidal configuration with bonding near the periphery and no bonding in a central zone as shown in Fig. $1[1,13]$.

The theory proposed by Chen [7] denied the role of other mechanisms such as melting, fusion, diffusion or mechanical interlocking in microelectronic applications. He pointed out that the most accepted explanation of the bond interface characteristics had been related to elasticity model proposed by Mindlin et
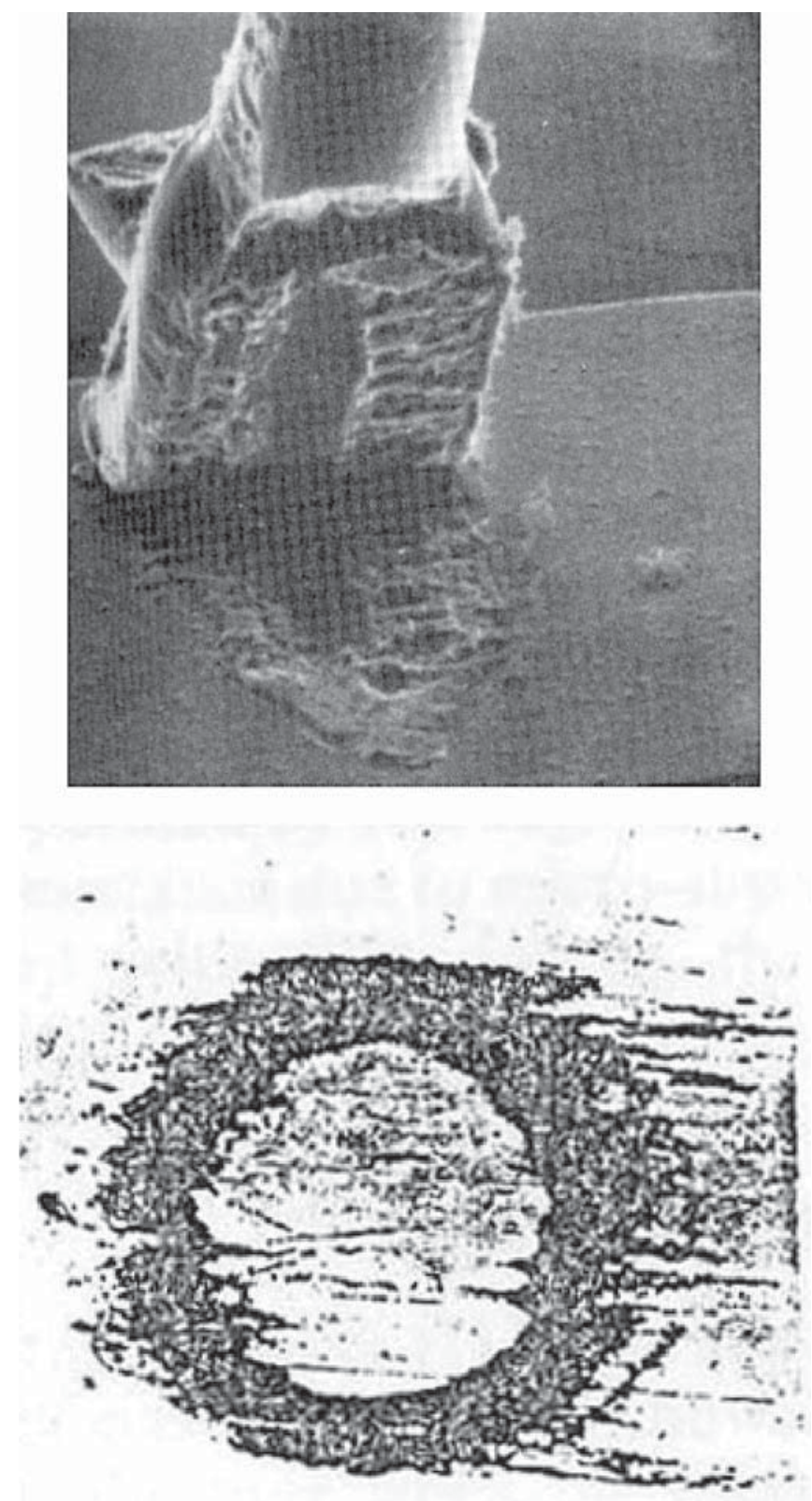

Fig. 1. The pattern of the bond and fretting caused by microslip (after Harman [1] and Johnson [13] respectively) al. [4], which was not specifically developed to account for ultrasonic application. Mindlin considered the contact between two spheres pressed into contact and analysed the relative elastic displacements between the spheres under the action of oscillating tangential load. In the proposed model, the central region was referred to as elastic or nonslip region, while the outer area had been represented as exhibiting slip, termed as microslip.

This interfacial form obtained from the above model simulated the commonly observed pattern of the ultrasonic bond where bonding predominated at the edge of the interfacial contact rather than at the centre. Therefore the conditions of microslip were used to account for the flow characteristics associated with the ultrasonic bond. Winchell and Berg [8] also found that the bonding area was an undeformed central area surrounded by a grooved peripheral region, which proved the role of microslip in the bonding process. The effects of the power, time and load on the features of the bond interface were shown qualitatively. However, their study was limited to the qualitative explanation of the bonding process.

Based on the literature available, the bonding process is a cyclic vibrating procedure with microslip between the gold ball and the die. Effort has been made to study the mechanism and modelling of the ultrasonic ball bonding process $[9,10]$. A microslip model is presented in this paper for the modelling and transient response. The purpose in formulating a model is to develop a conceptual understanding of how various bonding parameters work together to form the ultrasonic wire bond. The bonding process normally uses fixed parameters, i.e. a constant load with a particular power setting for a specific time. Therefore a model can provide a means for predicting the most probable parameter changes to improve bond reliability.

\section{Micro-slipping}

A schematic illustration of the bonding machine is shown in Fig. 2. The longitudinal vibration in the horn is transmitted to the gold ball through the capillary and becomes transverse vibration. When focused on the gold ball and die, the bonding process is modelled as microslip procedure. When a tangential force, whose magnitudes is less than the force of limiting friction, is applied to two bodies pressed into contact, it will not give rise to a sliding motion, but, nevertheless, will induce frictional traction at the contact interface.

The problem is illustrated in Fig. 3. Under the assumptions of elasticity and Amonton's law of friction, the normal force $f_{n}$ gives rise to a circular contact area with radius a and pressure distribution that is assumed to be uninfluenced by the existence of the tangential force $f_{t}$, hence to be given by the Hertz theory. The effect of the tangential force $f_{t}$ is to cause the body to deform in shear. When there is no slip between the two contact bodies under the tangential force, the tangential traction on the contact interface will be

$$
q(x)=\frac{f_{t}}{\pi\left(a^{2}-x^{2}\right)^{1 / 2}} .
$$




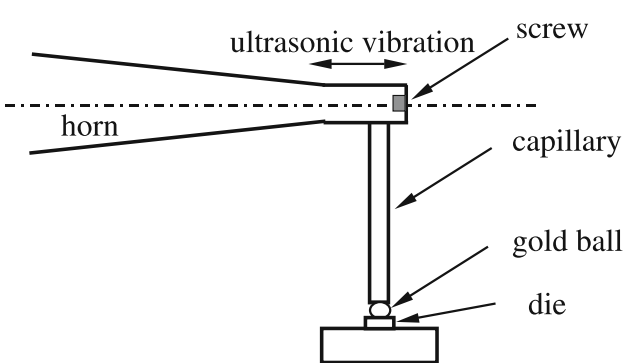

Fig. 2. Schematic illustration of the bonding tool and die pad
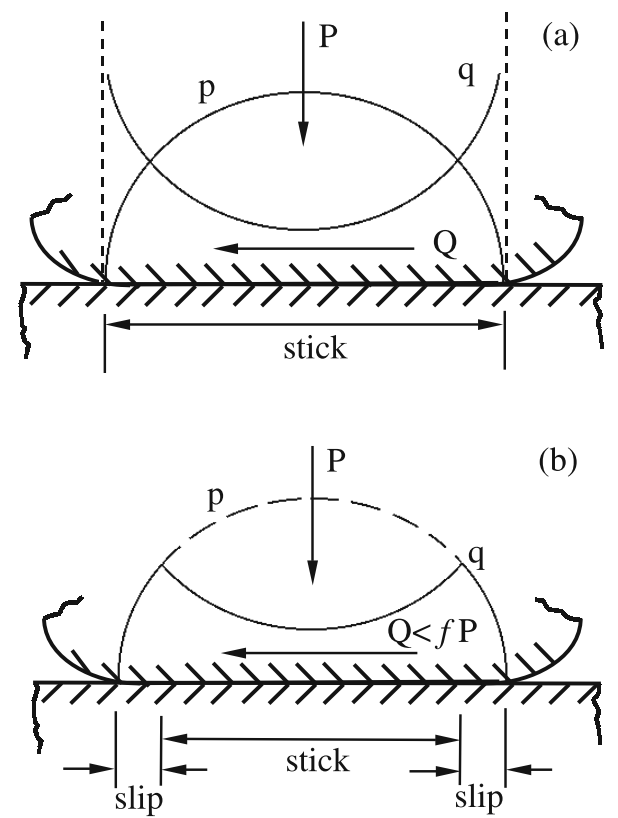

Fig. 3a,b. Traction distribution in contacting area with tangential force a without microslip b with microslip

It rises to a theoretically infinite value at the edges of the contact as shown in Fig. 3a. It is clear that this high tangential traction at the edge of the contact area can not be sustained, since that would require an infinite coefficient of friction. There must be some microslip as shown in Fig. $3 b$.

As indicated by the distorted centreline in Fig. 4, points on the contact surface will undergo tangential displacement $u_{x 1}$ relative to distant point $T_{1}$ in the undeformed region of the body. Clearly, if there is no sliding motion between the two bodies as a whole, there must be at least one point at the interface where the surface deforms without relative motion; but it does not follow that there is no slip anywhere within the contact area. In fact it will be shown that the effect of a tangential force less than the limiting friction force $\left(f_{t}<\mu f_{n}\right.$ where $\mu$ is the coefficient of friction) is to cause a small relative motion, referred to as 'slip' or 'microslip', over part of the interface. The remainder of the interface deforms without relative motion and in such regions the surfaces are said to 'adhere' or 'stick'.

To proceed with the analysis, the conditions of 'stick' and 'slip' must be considered. In Fig. 4, $A_{1}$ denotes a point on the interface that was coincident with point $O$ on the solid line before the application of the tangential force. $T_{1}$ denotes a point in the gold wire that is distant from the interface. $T_{1}$ was also on the solid line before the application of the tangential force. Under the action of the force, $T_{1}$ moves through effectively rigid displacement $\delta_{x 1}$ while $A_{1}$ experience tangential elastic displacement $u_{x 1}$ relative to $T_{1}$. If the absolute displacement $A_{1}$ (i.e. relative to $O$ ) is denoted by $s_{x 1}$, the components of slip can be written:

$s_{x 1}=\delta_{x 1}-u_{x 1}$

If the point $A_{1}$ is located in a 'stick' region, the slip $s_{x 1}$ will be zero so that $\delta_{x 1}=u_{x 1}$. At points inside a stick region the resultant tangential traction can not exceed its limiting value. Assuming Amonton's law of friction with a constant coefficient $\mu$, this restriction may be stated as:

$|q(x)| \leq \mu|p(x)|$

In a region where the surface slips, the tangential and normal traction are related by

$|q(x)|=\mu|p(x)|$.

During the application of the normal load there is no relative slip of points in contact. Subsequent application of a tangential force $f_{t}$ produces a slipped region. This slipped region is an annulus of constant outer radius $a$ and inner radius $c$ given by the expression [4]:

$c=a\left(1-\frac{f_{t}}{\mu f_{n}}\right)^{\frac{1}{3}}$.

The relative tangential displacement, $\delta$, of points in the two bodies distant from the interface is given by the equation:

$\delta=\frac{3(2-v) \mu f_{n}}{8 G a}\left[1-\left(1-\frac{f_{t}}{\mu f_{n}}\right)^{\frac{2}{3}}\right]$

where $G$ is shear modulus and $v$ is Poisson's ratio.

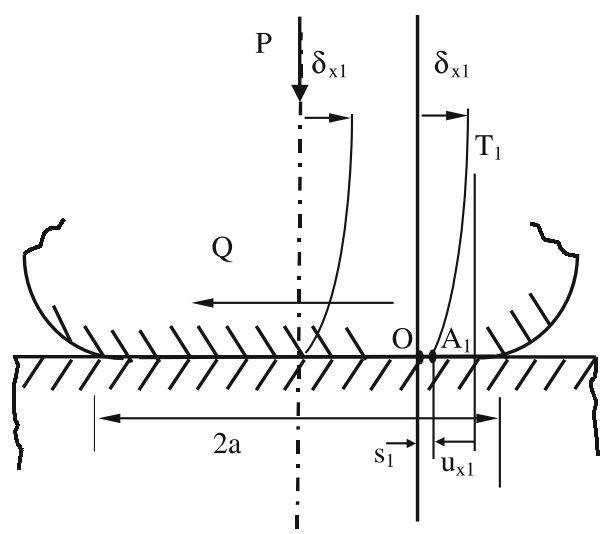

Fig. 4. Displacement components in stick and slip range during microslip 
As $f_{t}$ increases slip penetrates radially inward and when $f_{t}=\mu f_{n}=f_{t \text { max }}$ the entire contact area slips, a condition variously termed "slide" or "gross slip". In the theory and in the experiments to be described $f_{t}$ is restricted to a value $f_{t}^{*}<$ $f_{t \text { max }}$. During the initial loading interval, the displacement $\delta$ increases to a value $\delta^{*}$. During the unloading interval, $\delta=\delta_{d}$, the displacement-force relation given by Eq. 6 is modified and becomes:

$\delta_{d}=\frac{3(2-v) \mu f_{n}}{8 G a}\left[2\left(1-\frac{f_{t}^{*}-f_{t}}{2 \mu f_{n}}\right)^{\frac{2}{3}}-\left(1-\frac{f_{t}^{*}}{\mu f_{n}}\right)^{\frac{2}{3}}-1\right]$.

The modification is due to the presence of an initial state of stress and relative displacement at the outset of the unloading phase. On reloading $\delta=\delta_{i}=-\delta_{d}$. The difference between $\delta_{i}$ and $\delta_{d}$ implies that when $f_{t}$ oscillates between $\pm f_{t}^{*}$ a plot of the force-displacement $\left(f_{t}, \delta\right)$ diagram will yield a hysteresis loop of a particular shape. Being a dissipative process, microslip has the effect of making the state of contact stress dependent upon the history of loading.

Finally, the bonding process can be summarised as the following three steps:

1. Initial loading process:

$$
\begin{aligned}
\delta & =\frac{3(2-v)}{8 G a} \mu f_{n}\left(1-\left(1-\frac{f_{t}}{\mu f_{n}}\right)^{\frac{2}{3}}\right) \\
& =\frac{3(2-v)}{8 G a} \mu f_{n}\left(1-\frac{c^{2}}{a^{2}}\right)
\end{aligned}
$$

where $c=a\left(1-\frac{f_{t}}{\mu f_{n}}\right)^{\frac{1}{3}}$.

2. Unloading process:

$$
\begin{aligned}
\delta_{d} & =\frac{3(2-v)}{8 G a} \mu f_{n}\left(\frac{2 b^{2}}{a^{2}}-\frac{c^{2}}{a^{2}}-1\right) \\
& =\frac{3(2-v)}{8 G a} \mu f_{n}\left[2\left(1-\frac{f_{t}^{*}-f_{t}}{2 \mu f_{n}}\right)^{\frac{2}{3}}-\left(1-\frac{f_{t}^{*}}{\mu f_{n}}\right)^{\frac{2}{3}}-1\right]
\end{aligned}
$$

where $b=a\left(1-\frac{f_{t}^{*}-f_{t}}{2 \mu f_{n}} .\right)^{\frac{1}{3}}$

3. Reloading process:

$$
\begin{aligned}
\delta_{i} & =-\delta_{d}\left(-f_{t}\right) \\
& =-\frac{3(2-v) \mu f_{n}}{8 G a}\left[2\left(1-\frac{f_{t}^{*}+f_{t}}{2 \mu f_{n}}\right)^{\frac{2}{3}}-\left(1-\frac{f_{t}^{*}}{\mu f_{n}}\right)^{\frac{2}{3}}-1\right] .
\end{aligned}
$$

\section{Hysteric system}

It is clear that the microslip has a hysteretic characteristic between the oscillating force and displacement. The restoring force of a hysteretic system depends not only on the instantaneous displacement, but also on its past history.

The bilinear model proposed by Rogers and Boothroyd [11] is modified and used for wire bonder application in this paper. For the wire bonding process, a lumped microslip model is proposed, which is shown in Fig. 5a. The bonding tool, i.e. capillary, is modelled as two lumped masses with springs and dampers. The vibration introduced by the horn is represented by the movement of the base, and is denoted as $r \cos \omega t$. The interaction between the capillary and gold ball is modelled as a microslip procedure. The restoring force, i.e. bilinear hysteresis force, is represented as $f(x, \dot{x})$ and shown in Fig. 5b. The governing equation can be written as, (at first the damping is neglected for simplicity. It will be included later)

$m_{1} \ddot{x}_{1}+k_{1}\left(x_{1}-r \cos \omega t\right)+k_{2}\left(x_{1}-x_{2}\right)=0$

$m_{2} \ddot{x}_{2}+k_{2}\left(x_{2}-x_{1}\right)+f\left(x_{2}, \dot{x}_{2}\right)=0$

where $m_{1}$ and $m_{2}$ are concentrated mass, $k_{1}$ and $k_{2}$ are effective stiffness, $x_{1}$ and $x_{2}$ are displacements, while $r$ is the driving displacement from the horn, and $f(x, \dot{x})$ is the hysteric restoring force loaded on $m_{2}$ which is caused by microslip during bonding process.

The restoring force increases first along the straight line with the slope $K_{1}$. When the straight line meets the upper limit, the force increases then along another straight line with slope $K_{2}$ until the maximum displacement is accessed. Then the unloading process starts shown in Fig. 5b. The force decreases with slope $K_{1}$ until the lower limit. Then the slope changes to $K_{2}$ until the negative maximum displacement is accessed. The loading process then starts again. The area in the hysteresis loop is the

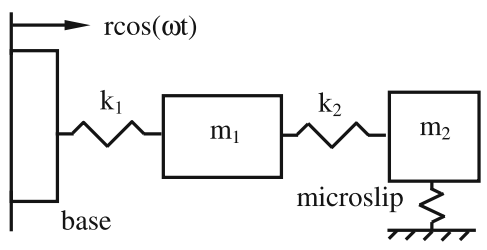

(a)

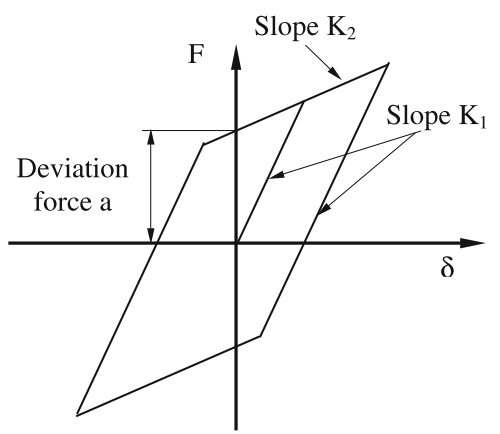

(b)

Fig. 5a,b. The microslip model of bonding process in ultrasonic wire bonders $\mathbf{a}$ the lumped mass model $\mathbf{b}$ the restoring force of microslip model 
energy dissipated in the bonding process. The governing Eqs. 11 and 12 are solved using MATLAB.

\section{Transient response results}

\subsection{Prediction}

Since a lumped mass-spring system is used to model the bonder components, a two degree-of-freedom system with $m_{1}=m_{2}$ and $k_{1}=k_{2}$ is assumed for simplicity. This is justified that the system yields a resonance at excitation frequency. However, a more accurate model of bonders using finite element method will be incorporated in [9]. The concentrated masses are calculated via the density of the capillary, $3960 \mathrm{~kg} / \mathrm{m}^{3}$ and the specification used in the wire bonder. The stiffness is selected to make the lower natural frequency of this two degree of freedom system be $60 \mathrm{kHz}$ and consequently another natural frequency is $157 \mathrm{kHz}$. The displacement $r$ is assumed to be $2 \times 10^{-6} \mathrm{~m}$, and $\omega$ is the angular frequency corresponding to the excitation frequency of $60 \mathrm{kHz}$. High frequency bonder can also be studied in a similar way.

The parameters for bilinear restoring force are difficult to determine. According to the references of Mindlin [4] and Johnson [5], two slopes are set as 7E5 and $2 \times 10^{5} \mathrm{~N} / \mathrm{m}$, respectively. The intersection between the straight line with slope of $2 \times 10^{5} \mathrm{~N} / \mathrm{m}$ and $\mathrm{y}$-axis is $20 \mathrm{~N}$. These three parameters need further experimental investigation, and they are related to the material properties of the gold wire and the connection between the gold wire and substrate.

After the determination of these parameters in the governing Eqs. 11 and 12 the transient results can be obtained. The velocity response in the time domain is shown in Fig. 6a, together with the phase plot, i.e. the displacement vs. velocity, shown in Fig. 6b. Other characteristics of the predicted response, the spectral content (FFT) of the signal is shown in Fig. 7, and the calculated hysteresis loop is shown in Fig. 8.

\subsection{Experimental evaluation}

It can be seen that the trend of the velocity response is very close to the typical bonding process signal measured elsewhere (e.g. Or et al. [12]), and an experiment was conducted from a typical bonder (Kulicke \& Soffa 4522 multi-process ball bonder) with $25 \mu \mathrm{m}$ gold wire (99.99\% gold with $2-6 \%$ elongation).

There are four parameters that can be varied in this machine to maintain the bonding quality, i.e. the bonding force, bonding power, bonding time and the bonding temperature. However, they are defined by a level setting on the wire bonder machine and the actual values of the parameters in setting for ultrasonic transducer are not known to end-users. In the experiment, the recommend parameters from Kulicke \& Soffa Company are used (power level $=2.5$, time setting $=6$, force level $=1.5$ and temperature $=150{ }^{\circ} \mathrm{C}$ ). It is believed that they are the usual parameters used in the industry. Parameters different from these may be used, such as different temperature.
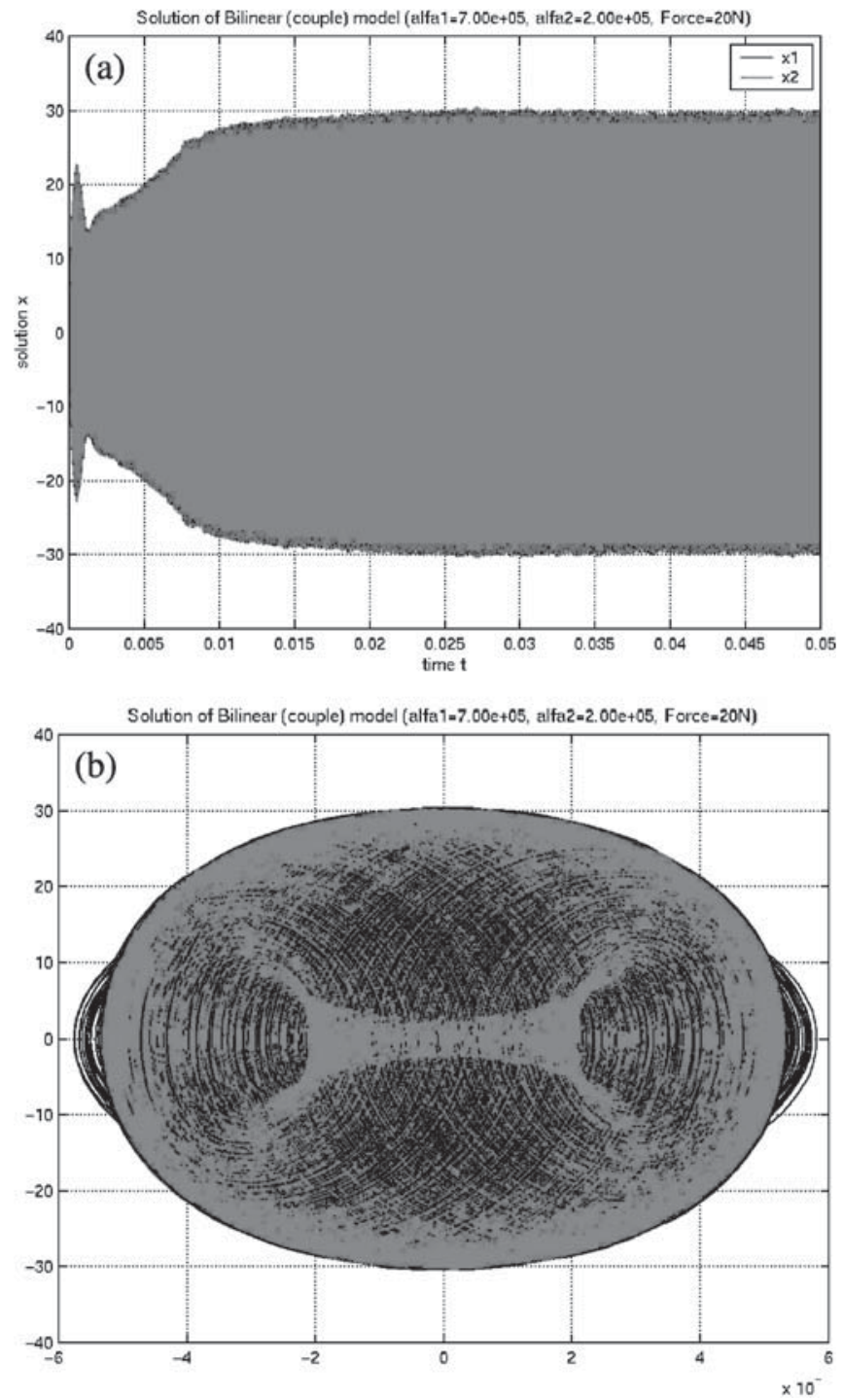

Fig. 6a,b. The calculated transient response at $\mathrm{m}_{1}$ using the microslip model a the velocity response; $\mathbf{b}$ the phase plot

The ultrasonic system in the bonder is a high-Q $60 \mathrm{kHz}$ transducer and PLL ultrasonic generator. The laser-vibrometer, OMETRON VPI Sensor, is used in the experiment to measure the velocity at the middle point of the capillary during the bonding process as shown in Fig. 9. The output signal of the laservibrometer is transmitted to a digital oscilloscope and saved in a computer.

The measured velocity response is shown in Fig. 10. It can be seen that the velocity oscillating amplitude first rises quickly to a maxima value and then decreases to a minima value. The velocity response then rises again but with a much slow rise-time, and it approaches a maximum, gradually decreases to a steady value. Depending on the bonding time setting, it would decrease to zero.

In comparison with the simulation result as shown in Fig. 6, the measured velocity amplitude is shown the same trend, with 


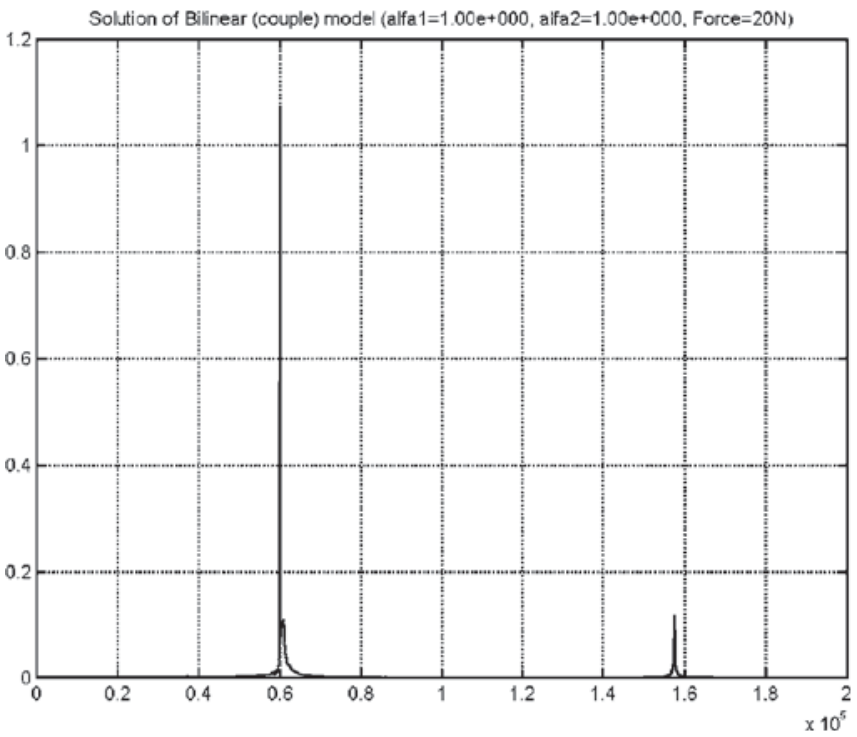

Fig. 7. The spectral content of the predicted transient response at $m_{1}$

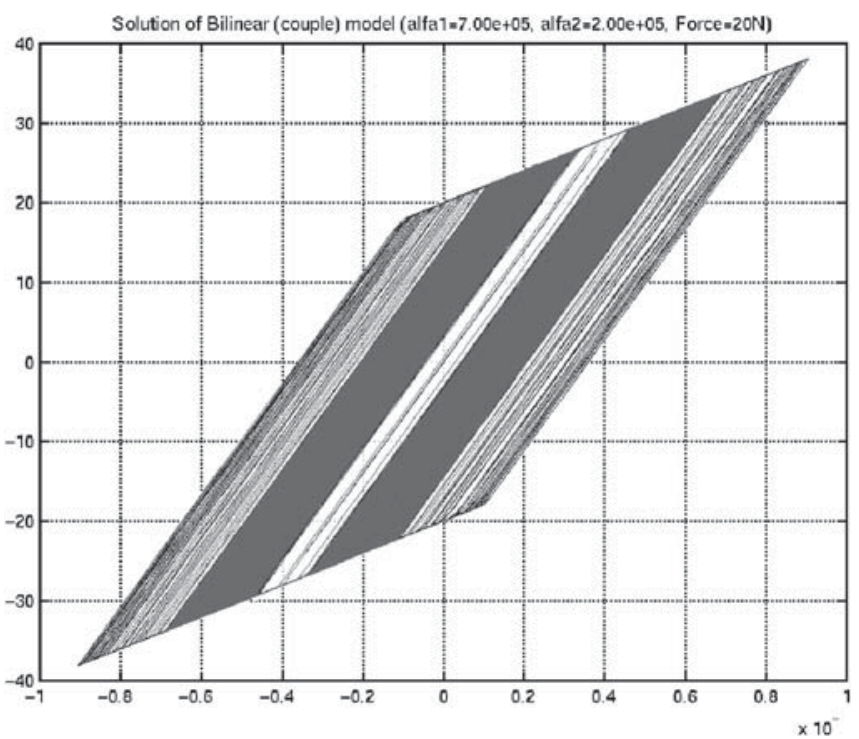

Fig. 8. The calculated hysteresis loop in the transient response at $m_{1}$

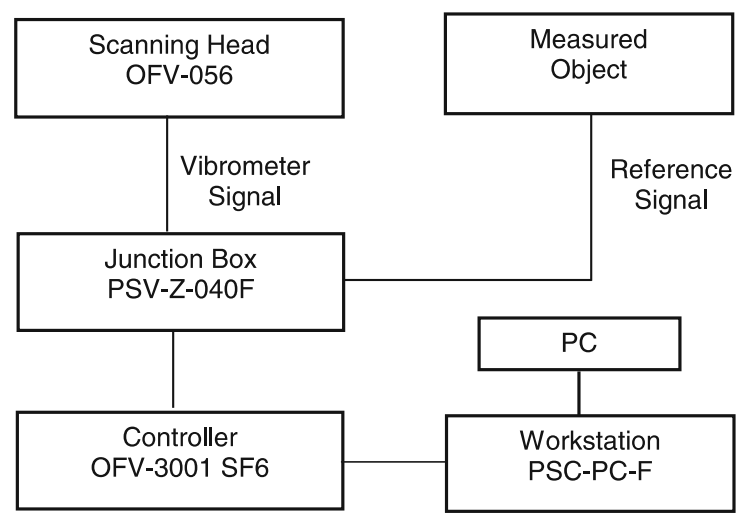

Fig. 9. Experiment set-up using a laser-vibrometer

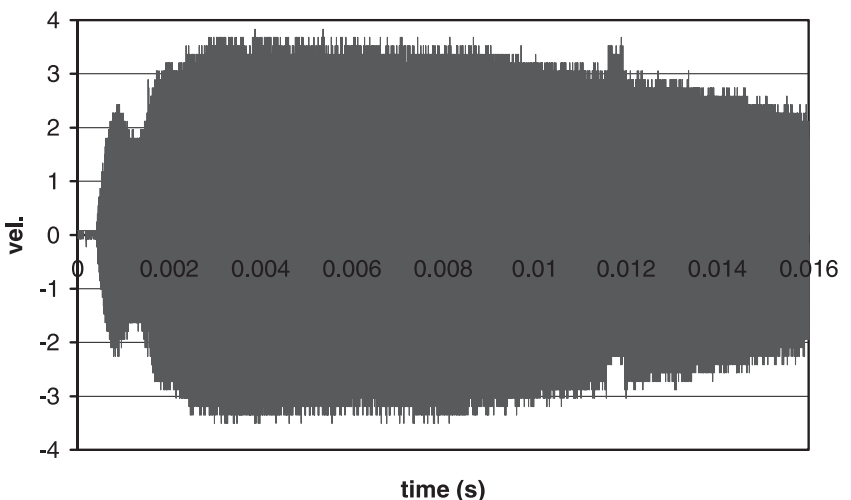

Fig. 10. The measured velocity response during the bonding process of a ultrasonic wire bonder by a laser-vibrometer

the same amplitude envelope, validating the theoretical model. The predicted velocity response finally settles at one constant amplitude and the hysteresis loop is steady along a fixed loop, which is represented by the outline of the red (or deeper) colour area with a maximum force of about $33 \mathrm{~N}$ as shown in Fig. 8.

Figure 11 shows the measured velocity of the bonder for varying bonding force, i.e. for large, medium and small bonding forces respectively, at the recommended bonding power setting (medium). It can be seen from Fig. 11 that with a higher bonding force setting, the measured velocity amplitude of the response is smaller. There is much less change in the shape of the response envelope if the bonding force is large, in comparison with that for smaller bonding force. Figure $11 \mathrm{~b}$ shows the measured response at the power and force settings recommended in the user's manual. The measured velocity shows a similar trend as the predicted response.

It can also be seen from the spectral content of the displacement response shown in Fig. 7 that there is a relatively small component of the signal at $157 \mathrm{kHz}$ besides the dominant component of $60 \mathrm{kHz}$. It is caused by the lack of damping in the governing equation. Usually, the solution of the vibration equation $m \ddot{x}+c \dot{x}+k x=f(t)$ includes two parts, i.e. the homogeneous solution $x_{h}$ for $m \ddot{x}+c \dot{x}+k x=0$ with resonant frequency and a particular solution $x_{s}$ at the excitation frequency. Usually the $x_{h}$ will diminish with time if there is damping. Therefore it is not considered in practice since there is always damping in practice. However, the components of resonant frequency will appear in the response if no damping is considered. The influence of $x_{h}$ will be discussed in detail in the following section on the steadystate response.

\section{Conclusions}

It is evident from the literature available that there are extensive studies on the bonding process achieved with an ultrasonic wire bonder, and they have provided a good understanding of the bonding mechanism. However, most of the studies are 

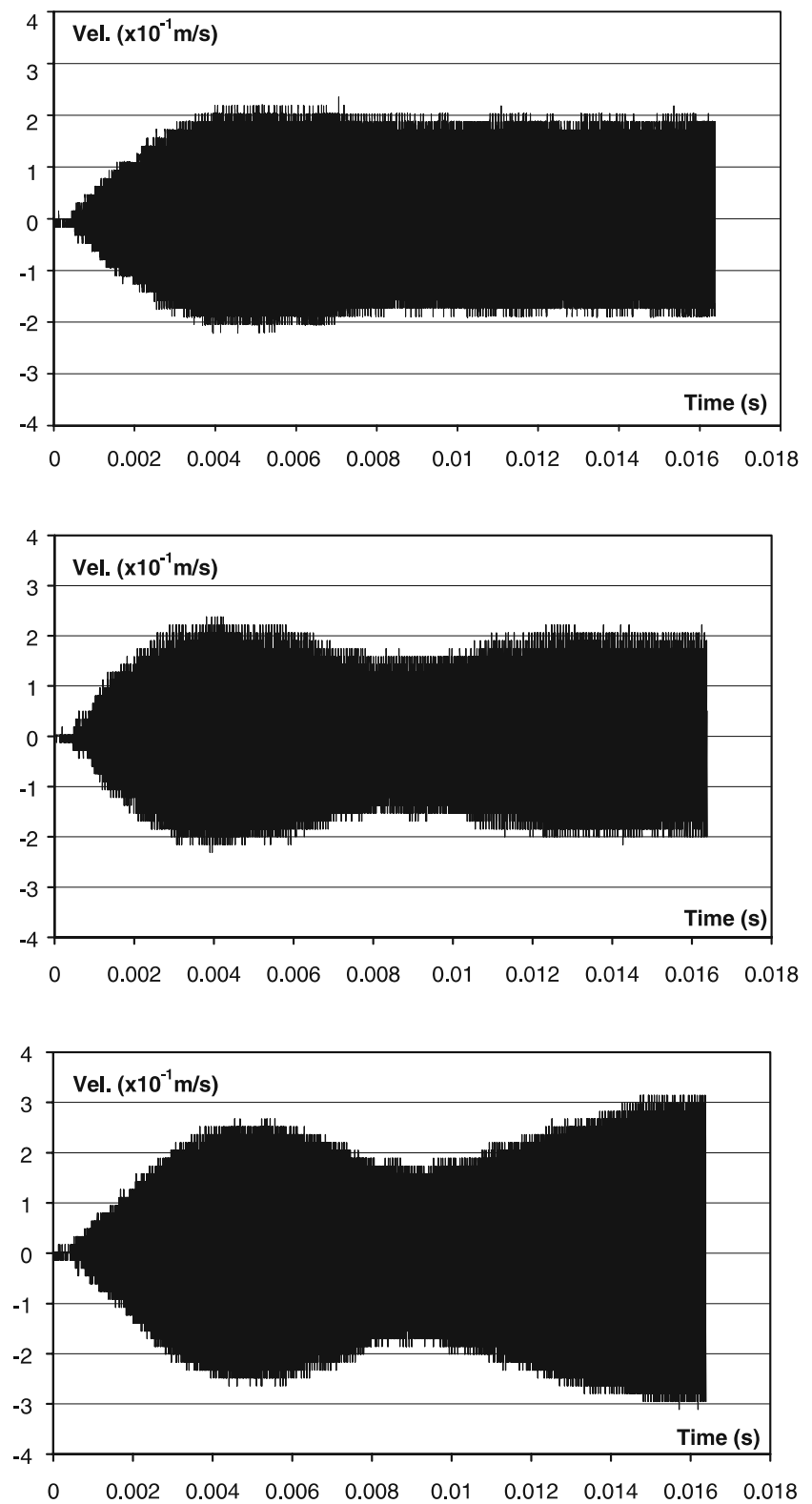

Fig. 11a-c. The measured velocity responses with different settings of bonding force a large $\mathbf{b}$ medium, and $\mathbf{c}$ small

empirically-based models and offer only a qualitative explanation. They are insufficient for further development of wirebonding technology. It is thus necessary to establish a mathematical model of the bonding process.

A microslip model for the bonding process is proposed in this paper based on the observation that the interface pattern is similar between the bond area and fretting area, which is largely caused by microslip. All of these phenomena have a toroidal pat- tern. In the bonding area, the centre zone is left unbonded and the bond occurs in the periphery area. In the fretting area caused by microslip, the periphery is fretted and the centre area is clear. It is assumed that during the bonding process, the periphery of the contact area between the gold ball and the die has relative movement and the oxide covering is abraded and thus the bond is made.

Based on this microslip model, the restoring force vs. displacement is assumed as a hysteresis loop. A bilinear hysteresis loop is assumed for simplicity. It can be seen that although it is very simple, the bilinear loop is enough to provide a similar trend between the predicted transient response and the measured signal of the bonding process. It can be seen that the velocity increases first and decreases when it reaches to certain value. After decreasing to the valley of the velocity, it increases steadily and finally the velocity becomes steady with larger value. However, it is also found that the parameters in the bilinear hysteresis loop are important for the response. Further study on its steady state response is reported elsewhere [10].

\section{References}

1. Harman G (1998) Wire bonding in microelectronics materials, processes, reliability, and yield, 2nd edn. McGraw-Hill, New York

2. Harman GG, Leedy KO (1972) An experimental model of the microelectronic ultrasonic wire bonding mechanism. 10th Ann Proc Reliab Phy Symp, pp 49-56

3. Joshi KC (1971) The formation of ultrasonic bonds between metals. Weld J pp 840-848

4. Mindlin RD, Mason WP, Osmer JF, Deresiewicz H (1952) Effects of an oscillating tangential force on the contact surfaces of elastic spheres. Proc 1st National Congress on Applied Mechanics, pp 203-208

5. Johnson KL (1954) Surface interaction between elastically loaded bodies under tangential forces. Proc Royal Soc, Series A 230:531-548

6. Wilson AD, Martin BD, Strope DH (1972) Holographic interferometry applied to motion studies of ultrasonic bonders. IEEE Trans on Sonics and Ultrasonics SU-19:453-461

7. Chen GKC (1972) The role of micro-slip in ultrasonic bonding of microelectronic dimensions. International Hybrid Microelectronics Symposium, 5-A-1-1-5-A-1-9

8. Winchell VH, Berg HM (1978) Enhancing ultrasonic bond development. IEEE Trans Compon, Hybrids, Manuf Tech CHMT-1:211-219

9. Hu CM, Guo N, Du H, Xu LM (2003) Dynamic characteristics of stacked piezoelectric transducers of ultrasonic wire- bonders used in integrated circuit packaging. Proc IME Part C: J Mech Eng Sci 217:341-352

10. Hu CM, Guo N, Du H, Jian XM, Chen M (in press) A microslip model of bonding process in ultrasonic wire bonders - Part II: steady state response. Int J Adv Manu Technol

11. Rogers PF, Boothroyd G (1975) Damping at metallic interfaces subjected to oscillating tangential loads. Trans ASME: J Eng Ind August:1087-1093

12. Or SW, Chan HLW, Lo VC, Yuen CW (1998) Ultrasonic wire-bond quality monitoring using piezoelectric sensor. Sensor Actuat A 65:69-75

13. Johnson KL (1982) One hundred years of Hertz Contact. Proc Instn Mech Engrs 196:363-378 